\title{
¿POR QUÉ LAS MUJERES NO SE TOMAN EL PAPANICOLAOU? RESPUESTAS ENTREGADAS POR LOS PROFESIONALES DEL PROGRAMA CÁNCER CERVICOUTERINO - AUGE DEL SERVICIO DE SALUD METROPOLITANO SUR ORIENTE *
}

\author{
María Teresa Urrutia S. ${ }^{a}$ PhD, Alejandra Araya G. ${ }^{a}$, Lauren Poupin B. ${ }^{b}$ \\ a Enfermera Matrona, Facultad de Medicina, Escuela de Enfermería, Departamento de Salud de la Mujer, Universidad \\ Católica de Chile. ${ }^{b}$ Enfermera Matrona, Magister en Salud Pública.
}

\footnotetext{
* Esta investigación forma parte del proyecto FONIS (Fondo Nacional de Investigación en Salud) SA05I20047, CONICYT, MINSAL, Chile.
}

\section{RESUMEN}

Antecedentes: Los beneficios del test de Papanicolaou (Pap) en reducir la mortalidad de cáncer cervicouterino han sido bien documentados a nivel mundial y nacional. Sin embargo, aún persisten mujeres que no se realizan el Pap rutinariamente. Objetivo: Conocer la percepción que tienen los profesionales de la salud en relación a las causas por las cuales las mujeres no se toman la prueba del Pap. Método: Estudio descriptivo transversal, con una muestra de 59 profesionales del Servicio de Salud Metropolitano Sur-Oriente de la ciudad de Santiago, provenientes de los tres niveles de atención del programa cáncer cervicouterino AUGE. Resultados: Promedio de edad de los profesionales fue de 39,2 $\pm 10,3$ años. El tiempo promedio en el cargo es de $7,7 \pm 7,4$ años. Un $41 \%$ de los profesionales de la salud tienen la percepción que las mujeres tienen un conocimiento parcial sobre la importancia de realizarse el Pap, mientras que un $48,3 \%$ refiere que las mujeres no tienen conocimiento sobres las normas de control de Pap. Falta de conocimiento, miedo, dejación y la falta de tiempo por parte de las mujeres surgen como las principales razones que los profesionales atribuyen a que las mujeres no se realicen el Pap. Conclusiones: Los resultados de este estudio pueden servir para diseñar y/o actualizar los contenidos educativos entregados a las mujeres con el propósito de aumentar el conocimiento y el nivel de conciencia sobre la importancia de la toma de esta prueba.

\section{PALABRAS CLAVE: Papanicolaou, barreras, creencias}

\section{SUMMARY}

Background: Benefits of screening programs using Papanicolaou test (Pap test) reducing cervical cancer related mortality has been documented worldwide and at national level, in Chile. However, there are women who do not have a Pap test routinely. Objective: To know the perception of health care providers about the reasons why some women do not have a Pap smear. Method: Cross sectional study with a sample of 59 health care providers from the Servicio de Salud Metropolitano Sur-Oriente in Santiago, Chile; representing all the three different levels of care of the national health program called AUGE. Results: Health care providers were in average $39 \pm 10$ years old, with an average of $7.7 \pm 7$ years working in the health service. A $41 \%$ of the health care providers have the perception that women had a partial knowledge about the importance of having a Pap smear; while a $48.3 \%$ of them refer that women do not have knowledge related to the Pap 
smear guidelines. Lack of knowledge, fear, neglect by women, and lack of time emerged as the principal reasons why women do not have a Pap smear based on health care provider's perception. Conclusions: The results from this research can help in designing and/or actualizing educative contents for women with the objective of increase the level of knowledge and the awareness about the importance in have a Pap smear.

\section{KEY WORDS: Papanicolaou, barriers, beliefs}

\section{INTRODUCCIÓN}

Los beneficios del Papanicolaou (Pap) para reducir la mortalidad del cáncer cervicouterino (CC) han sido bien documentados a nivel internacional (1-3) y nacional (4-6). El Pap es un método eficiente, asequible y efectivo en la detección de cambios en la citología del cuello uterino; está ampliamente disponible y es utilizado rutinariamente en la mayoría de los centros de salud (7). Chile inició el programa de pesquisa y control del CC en los años 70 (8), tras lo cual la mortalidad por esa patología ha sido reducida (4-6).

Aunque el tamizaje regular con el Pap ha sido comprobado como una estrategia efectiva de prevención de $\mathrm{CC}$, todavía existen mujeres que no recurren rutinariamente a este examen (7). El objetivo de este estudio es conocer la percepción que tienen los profesionales de la salud del programa AUGE-CC, en relación a las causas por las cuales las mujeres no se toman del Pap.

\section{MATERIAL Y MÉTODO}

Estudio descriptivo de corte transversal en un grupo de profesionales de la salud del Servicio de Salud Metropolitano Sur Oriente (SSMSO). La muestra está constituida por profesionales del programa AUGE-CC de los tres niveles de atención: en el nivel primario por el profesional matrón(a) responsable del programa de cada consultorio, en el nivel secundario por el médico y matrón(a) del policlínico de la especialidad de cada centro hospitalario (Complejo Asistencial Dr. Sótero del Río y Hospital Padre Alberto Hurtado), y en el nivel terciario por el médico y matrón(a) de los servicios de ginecología oncológica de los dos centros hospitalarios, y los profesionales del Instituto Nacional del Cáncer, donde se realiza el tratamiento de radioterapia a algunas de las usuarias con lesión invasora. La muestra corresponde a un total de 59 profesionales de la salud, que equivale al $98 \%$ del universo (solo 1 profesional no contestó el cuestionario). Los criterios de inclusión fueron: ser profesional del área de la salud que trabaje en los centros hospitalarios señalados o consultorios respectivos, estar en atención directa con la usuaria del programa AUGE-CC y estar en ejercicio del cargo por lo menos 6 meses previo a la aplicación del cuestionario. La recolección de los datos fue realizada durante el periodo comprendido entre mayo y octubre de 2006. Las variables medidas fueron: características de los profesionales (edad, profesión, especialidad, lugar de trabajo y tiempo en el cargo), percepción del conocimiento que tienen las usuarias acerca de la importancia de realizarse el Pap (escala de Likert de tres alternativas), y de la norma de control de Pap (escala de Likert de tres alternativas), y las causas por las cuales las mujeres no se realizan el Pap (pregunta abierta). Para el análisis de esta última pregunta se utilizó análisis de contenido según el método descrito por Krippendorf (9), generando una descripción temática de las respuestas entregadas por los profesionales. Esta investigación contó con la aprobación de los comités de ética: de la Escuela de Enfermería de la Pontificia Universidad Católica de Chile y del SSMSO. Se solicitó firmar voluntariamente el consentimiento informado a cada uno de los profesionales involucrados. Los datos se analizaron con el programa SPSS 16.0 y se utilizaron medidas de tendencia central, de dispersión y análisis de frecuencia.

\section{RESULTADOS}

Características de los profesionales. El promedio de edad de los profesionales fue de 39,2 \pm 10,3 años, con un rango de 25 a 70 años. Del total de la muestra, 45 son matronas (es), 13 médicos y 1 enfermera. El $69,5 \%$ trabaja en el nivel primario, el $6,8 \%$ en el nivel secundario, el $3,4 \%$ en el nivel secundario y terciario, y el $20,4 \%$ en el nivel terciario. El tiempo promedio en el cargo es de 7,7 $\pm 7,4$ años con un rango de 6 meses a 34 años. El 22\% de la muestra refiere tener una especialización en oncología, con un período de formación entre 1,5 a 4 años; todos ellos corresponden a profesionales médicos.

Conocimiento de las mujeres según los profesionales que les brindan atención. Un $41,4 \%$ de los profesionales de la salud tiene la percepción que las mujeres tienen un conocimiento parcial sobre la 
importancia de realizarse el Pap, un 29,3\% de ellos señala que las mujeres no tienen conocimiento y el $29,3 \%$ restante refiere que las mujeres si tienen conocimiento sobre la importancia de tomarse la prueba de Pap.

Un $48,3 \%$ de los profesionales entrevistados señala que las mujeres no tienen conocimiento sobre la norma de control de Pap, un $32,8 \%$ de ellos refiere que las mujeres tienen un conocimiento parcial y el 18,9\% restante señala que las mujeres si tienen conocimiento sobre la norma de Pap.

Frente a la pregunta abierta ¿a qué atribuye usted que las mujeres no se tomen el test del Pap?, surgieron los siguientes temas en el análisis de contenido: falta de conocimiento con un $45,6 \%$, miedo con un $38,6 \%$, dejación por parte de la mujer con un $29,8 \%$ y problemas atribuidos al tiempo de las mujeres con un $22,8 \%$. El desglose de los subtemas de cada uno de estos temas se encuentra detallado en la Tabla I.

\section{DISCUSIÓN}

Los esfuerzos por aumentar el número de mujeres que se practican rutinariamente la prueba de
Pap debieran estar centrados en dos grandes aspectos: potenciar el conocimiento de las mujeres sobre el Pap y mejorar las influencias del entorno que limitan a las mujeres a realizarse el examen (3). En este sentido, esta investigación entrega información importante sobre la percepción de un grupo de profesionales de la salud, que trabaja directamente con estas mujeres, en torno a cuáles son las causas por las cuales ellos creen que las mujeres no se realizan el Pap.

Llama la atención que el sub-tema que más frecuentemente emergió del análisis de contenido es la percepción de los profesionales sobre la falta de conocimiento de las mujeres, resultado que concuerda con las principales razones por las cuales las mujeres chilenas refieren no tomarse el Pap (10). Esta convergencia en los resultados, por dos fuentes distintas, nos lleva a pensar que este es un problema real, que necesita ser abordado, para incrementar los niveles de conocimiento sobre la importancia del Pap en las mujeres que asisten al consultorio, para establecer un mayor énfasis, en aquella población que no se realiza el Pap. Falta de conocimiento de las mujeres, también ha sido reportada por otros investigadores. Las mujeres

Tabla I

\section{PERCEPCIÓN DE LOS PROFESIONALES DE LA SALUD ACERCA DE LAS CAUSAS POR LAS CUALES LAS MUJERES NO SE REALIZAN EL TEST DE PAPANICOLAOU $(n=59)$ *}

\begin{tabular}{lcc}
\hline Sub-temas & Temas & Respuestas (\%) \\
\hline $\begin{array}{l}\text { a. Falta de información disponible para las mujeres } \\
\text { b. Falta de educación a las mujeres }\end{array}$ & Falta de conocimiento & 45,6 \\
c. Desconocimiento de los beneficios de la toma del Pap & \\
d. Problemas con el procedimiento (vergüenza, pudor, dolor) & \\
e. Creencias erradas en torno a la toma del Pap &
\end{tabular}
a. Al diagnóstico de cáncer
Miedo
b. Al tener un Pap alterado
c. A la amonestación de los profesionales de la salud
d. A la movilización del dispositivo intrauterino
a. Olvido
b. Falta de interés
c. Mujeres no quieren ir al consultorio
d. Postergación por familiares y el hogar
e. Falta de autocuidado
f. Falta de preocupación

Dejación

a. Horarios de atención no compatible con mujeres trabajadoras

Tiempo

b. Falta de disponibilidad de horas de atención en el consultorio

c. Falta de tiempo de la mujer

* El porcentaje final es más del 100\% ya que cada profesional pudo haber dado más de una respuesta. 
refieren tener un bajo entendimiento sobre lo que significa la prueba del Pap o confunden los términos de la prueba de Papanicolaou con el examen pélvico (11-17). Malentendidos sobre el significado de la prueba del Pap tienen el potencial de dificultar la comunicación entre los profesionales de la salud y las usuarias (13).

Falta de conocimiento asociado al procedimiento tales como: vergüenza, pudor, dolor, también han sido descrito por otros investigadores $(17,18)$, abriendo una línea importante de educación a las mujeres en torno al procedimiento de la toma del Pap, de manera de desmitificar creencias en torno al procedimiento que puedan estar obstaculizando que las mujeres acudan a realizarse el Pap. Los centros de salud junto con los profesionales que atienden a las mujeres deben ser sensibles para prevenir y evitar situaciones de incomodidad y vergüenza hacia las usuarias $(10,19)$.

En este estudio, el segundo sub-tema reportado por los profesionales fue el miedo, coincidiendo con otros estudios en donde el miedo ha sido reportado como una de las razones explícitas que las mujeres refieren para no tomarse el Pap (7). Por ejemplo, el miedo de las mujeres a recibir un diagnóstico de cáncer ha sido descrito por otros autores $(15,16)$. Sin embargo, cabe destacar que la amonestación por parte de los trabajadores de la salud es uno de los subtemas relacionado al miedo. Un cuidado en salud sensible hacia las mujeres, que otorgue una consejería sobre la importancia de realizar el Pap, es un aspecto necesario a considerar para incentivar a las mujeres a la realización de este examen (20).

Postergación de las mujeres, que priorizan a sus familiares y a la atención del hogar, en detrimento del propio cuidado, es un sub-tema que emergió dentro del tema de la dejación de las mujeres. Los compromisos derivados del cuidado de niños es una de las razones explícitas que las mujeres refieren para no realizarse el Pap (7). Medidas destinadas a disminuir los tiempos de espera de las mujeres son claves para que éstas puedan acercarse a los centros de salud de una manera simple y rápida, de manera que el cuidado de familiares no se convierta en una barrera para tomarse el Pap.

La falta de tiempo, fue uno de los subtemas que emergió dentro del análisis de contenido, como una de las causas por las cuales las mujeres no se realizan el Pap. Los horarios de atención no compatibles con los de las mujeres trabajadoras, y la falta de disponibilidad de horas de atención en el consultorio fueron razones sugeridas por los profesionales que trabajan en el programa AUGE-CC por la cual mujeres chilenas no se toman el Pap.
Esta barrera también ha sido reportada por otros investigadores. Por ejemplo, la falta de flexibilidad en el horario de atención de los centros de la salud es una de las barreras por las cuales mujeres mexicanas no se toman el Pap (21). La reducción de los tiempos de espera cuando las mujeres son citadas a tomarse el examen $(15,22,23)$, la facilidad de acceso a las horas de atención (20), y el acomodar las agendas de citación de los consultorios a los horarios en los cuales es más factible que las mujeres puedan acceder a realizarse el Pap (24),son medidas que pueden ser implementadas para capturar un grupo de mujeres que por estas razones no se está realizando rutinariamente el examen o para aquel grupo que no se lo ha tomado nunca. El mejoramiento de las funciones administrativas de los centro de salud, tales como la citación de los pacientes o la notificación de ellos puede ayudar a disminuir las barreras de la accesibilidad a los centros de salud (7).

Falla en el cumplimiento de las guías de tamizaje del programa AUGE-CC es una de las preocupaciones de la atención primaria de salud (25). Países como Chile e Israel, han desarrollado programas de salud con coberturas universales para hacer la atención primaria más accesible a todos los grupos socioeconómicos $(26,27)$, sin embargo, este tipo de sistema no ha eliminado los reportes de dificultades en el acceso a la atención de salud (28).

Los resultados de esta investigación en conjunto con la información extraída de las mujeres (10) debieran ser incorporados para actualizar y/o desarrollar intervenciones educativas efectivas en este grupo de mujeres que aborden principalmente la falta de conocimiento de este grupo así como también sus miedos (29). Estrategias efectivas utilizadas para aumentar la adherencia al Pap combinan campañas educativas masivas en los medios de comunicación, junto con la entrega de información y educación individual diseñada específicamente para las mujeres (30). Materiales educativos de fácil lectura y evaluados en la población de mujeres en las cuales serán entregados $(7,12)$ son claves para facilitar la educación de esta población objetivo. Las campañas educativas tienen como propósito aumentar el conocimiento y la conciencia de la importancia de la toma de Pap (30). Los profesionales de la salud debieran estar consientes de la falta de conocimiento que las mujeres tienen en torno al Pap y ajustar la consejería que ellos proveen a las mujeres durante la atención de salud (12).

En este contexto, el fortalecimiento de la educación a las mujeres en el control ginecológico es otro punto importante a considerar. Por ejemplo, comprobar la comprensión de las mujeres sobre los 
contenidos educativos que se les han entregado, usando estrategias tales como: la devolución de los contenidos, haciendo que las mujeres describan en sus propias palabras los contenidos entregados (12), son herramientas que los profesionales de la salud pueden utilizar para reforzar los contenidos educativos sobre la importancia de realizarse el Pap.

En este sentido, la participación de los profesionales de la salud es clave para incrementar el número de mujeres que se realizan el Pap (31). La recomendación directa por parte de los profesionales de realizarse el Pap ha demostrado ser uno de los factores más influyentes en la decisión de las mujeres a realizarse este examen (19,32-34). Otros profesionales de salud, además de los del área ginecológica, debieran sentirse más involucrados en reforzar estas medidas preventivas al momento de dar atención de salud (27). En este sentido estudios anteriores han sugerido que la especialidad de los médicos puede influir en el número de mujeres que se realiza el Pap (35).

Futuros estudios deben apuntar a incrementar el entendimiento de los factores que influyen en la adherencia a la toma del Pap desde la perspectiva de las mujeres $(7,29)$, junto con conocer los factores que influyen en la accesibilidad de los centros médicos de atención primaria $(29,36)$. Otra pregunta que queda abierta es saber si las mujeres que no se realizan el Pap son mujeres cercanas al sistema de salud, ya que la educación sobre prevención de CC debiera involucrar a todas las mujeres y no solo a aquellas que asisten al sistema de salud (37).

\section{CONCLUSIÓN}

Los resultados de este estudio pueden servir para diseñar y/o actualizar los contenidos educativos entregados a las mujeres con el propósito de aumentar el conocimiento y el nivel de conciencia sobre la importancia de la toma de esta prueba.

\section{BIBLIOGRAFÍA}

1. Fletcher SW, Harris RP, Gonzalez JJ, Degnan D, Lannin DR, Strecher VJ, et al. Increasing mammography utilization: a controlled study. J Natl Cancer Inst 1993;85(2):112-20.

2. Dewar MA, Hall K, Perchalski J. Cervical cancer screening. Past success and future challenge. Prim Care 1992;19(3):589-606.

3. Taylor VM, Nguyen TT, Jackson JC, McPhee SJ. Cervical cancer control research in Vietnamese American communities. Cancer Epidemiol Biomarkers Prev 2008;17(11):2924-30.
4. Capurro I, Rojas J, Pino T, Vásquez C, Garay J, Venegas M. Programa de detección y control de cáncer de cuello uterino en Servicio Salud Araucanía Sur. Rev Chil Obstet Ginecol 2002;6(2):114-20.

5. Guzmán S, Salas P, Puente R, Hott H, Israel E, Guzmán $\mathrm{R}$. Pesquisa y control del cáncer cérvico-uterino en el Servicio de Salud de Valdivia (1993-2003). Rev Méd Chile 2005;133(6):685-92.

6. Donoso E, Cuello M, Villarroel L. Reducción de la mortalidad por cáncer cérvico uterino en Chile, 19902003. Rev Chil Obstet Ginecol. 2006;71(5):307-12.

7. Abercrombie PD. Improving adherence to abnormal Pap smear follow-up. J Obstet Gynecol Neonatal Nurs 2001;30(1):80-8.

8. Sankaranarayanan R, Budukh AM, Rajkumar R. Effective screening programmes for cervical cancer in low- and middle-income developing countries. Bull World Health Organ 2001;79(10):954-62.

9. Krippendorf K. Content Analysis. An Introduccion to its methodology: Thousands Oaks: Sage Publications 2004.

10. Urrutia MT, Poupin L, Concha X, Viñales D, Iglesias $\mathrm{C}$, Reyes V. ¿Por qué las mujeres no se toman el Papanicolau?: barreras percibidas por un grupo de mujeres ingresadas al programa de cáncer cervicouterino AUGE. Rev Chil Obstet Ginecol 2008;73(2):98-103.

11. McFarland DM. Cervical cancer and Pap smear screening in Botswana: knowledge and perceptions. Int Nurs Rev 2003;50(3):167-75.

12. Fortner KB, Zite NB, Wallace LS. In my own words: misunderstanding of Pap smears and colposcopy among Appalachian women. J Low Genit Tract Dis 2007;11(4):251-7.

13. Blake DR, Weber BM, Fletcher KE. Adolescent and young adult women's misunderstanding of the term Pap smear. Arch Pediatr Adolesc Med 2004;158(10):966-70.

14. McMullin JM, De Alba I, Chavez LR, Hubbell FA. Influence of beliefs about cervical cancer etiology on Pap smear use among Latina immigrants. Ethn Health 2005;10(1):3-18.

15. Byrd TL, Chavez R, Wilson KM. Barriers and facilitators of cervical cancer screening among Hispanic women. Ethn Dis 2007;17(1):129-34.

16. Smith M, French L, Barry HC. Periodic abstinence from Pap (PAP) smear study: women's perceptions of Pap smear screening. Ann Fam Med 2003;1(4):203-8.

17. Ho V, Yamal JM, Atkinson EN, Basen-Engquist K, Tortolero-Luna G, Follen M. Predictors of breast and cervical screening in Vietnamese women in Harris County, Houston, Texas. Cancer Nurs 2005;28(2):119-29.

18. Lee MC. Knowledge, barriers, and motivators related to cervical cancer screening among Korean-American women. A focus group approach. Cancer Nurs 2000;23(3):168-75. 
19. Bazargan M, Bazargan SH, Farooq M, Baker RS. Correlates of cervical cancer screening among underserved Hispanic and African-American women. Prev Med 2004;39(3):465-73.

20. Johnson CE, Mues KE, Mayne SL, Kiblawi AN. Cervical cancer screening among immigrants and ethnic minorities: a systematic review using the Health Belief Model. J Low Genit Tract Dis 2008;12(3):232-41.

21. Hunt LM, de Voogd KB, Akana LL, Browner CH. Abnormal Pap screening among Mexican-American women: impediments to receiving and reporting follow-up care. Oncol Nurs Forum 1998;25(10):1743-9.

22. Wiesner-Ceballos C, Vejarano-Velandia M, CaicedoMera JC, Tovar-Murillo SL, Cendales-Duarte R. [Cervical cytology in Soacha, Colombia: social representation, barriers and motivation]. Rev Salud Publica (Bogota) 2006;8(3):185-96.

23. Kahn JA, Chiou V, Allen JD, Goodman E, Perlman SE, Emans SJ. Beliefs about Papanicolaou smears and compliance with Papanicolaou smear follow-up in adolescents. Arch Pediatr Adolesc Med 1999;153(10):1046-54.

24. Valenzuela M, Miranda A. ¿Por qué no me hago el Papanicolau? Barreras psicológicas de mujeres de sectores populares de Santiago de Chile. Rev Chil Salud Publica 2001;5(2-3):75-80.

25. Murff HJ, Gandhi TK, Karson AK, Mort EA, Poon EG, Wang SJ, et al. Primary care physician attitudes concerning follow-up of abnormal test results and ambulatory decision support systems. Int J Med Inform 2003;71(2-3):137-49.

26. Ministerio de Salud de Chile. Guía Clínica Cáncer Cervicouterino. 3a ed: Santiago: Minsal, 2006.

27. Benyamini $Y$, Blumstein $T$, Boyko V, Lerner-Geva L. Cultural and educational disparities in the use of primary and preventive health care services among midlife women in Israel. Womens Health Issues 2008;18(4):257-66.

28. Schoen C, Simantov E, Gross R, Brammli S, Leiman J. Disparities in women's health and health care experiences in the United States and Israel: findings from 1998 National Women's Health Surveys. Women Health 2003;37(1):49-70.

29. Selvin E, Brett KM. Breast and cervical cancer screening: sociodemographic predictors among White, Black, and Hispanic women. Am J Public Health 2003;93(4):618-23.

30. Black ME, Yamada J, Mann V. A systematic literature review of the effectiveness of community-based strategies to increase cervical cancer screening. Can J Public Health 2002;93(5):386-93.

31. Harpole LH, Mort EA, Freund KM, Orav J, Brennan TA. A comparison of the preventive health care provided by women's health centers and general internal medicine practices. J Gen Intern Med 2000;15(1):1-7.

32. Caplan LS, Wells BL, Haynes S. Breast cancer screening among older racial/ethnic minorities and whites: barriers to early detection. J Gerontol 1992;47 Spec No:101-10.

33. Camirand J, Potvin L, Beland F. Pap recency: modeling women's characteristics and their patterns of medical care use. Prev Med 1995;24(3):259-69.

34. Bazargan M, Bazargan SH, Calderon JL, Husaini BA, Baker RS. Mammography screening and breast selfexamination among minority women in public housing projects: the impact of physician recommendation. Cell Mol Biol (Noisy-le-grand). 2003;49(8):1213-8.

35. Haggstrom DA, Phillips KA, Liang SY, Haas JS, Tye $\mathrm{S}$, Kerlikowske K. Variation in screening mammography and Papanicolaou smear by primary care physician specialty and gatekeeper plan (United States). Cancer Causes Control 2004;15(9):883-92.

36. Coughlin SS, Uhler RJ, Blackman DK. Breast and cervical cancer screening practices among American Indian and Alaska Native women in the United States, 1992-1997. Prev Med 1999;29(4):287-95.

37. Somkin CP, Hiatt RA, Hurley LB, Gruskin E, Ackerson $L$, Larson $P$. The effect of patient and provider reminders on mammography and Papanicolaou smear screening in a large health maintenance organization. Arch Intern Med 1997;157(15):1658-64. 